Conclusions Similar to adolescents, burden of S\&R injury is high in children. Children with pDCD and/or pADHD were not at a greater risk of S\&R injury than typically developing children. Injury prevention strategies should target children and adolescents.

\section{SPORT-RELATED INJURY IN HIGH SCHOOL STUDENTS: CHECKING IN AFTER A DECADE OF INJURY PREVENTION INTERVENTIONS}

\begin{abstract}
1,2,3Amanda M Black, 'Derek Meeuwisse, 1,2,3Paul H Eliason, 1,2,3,4,5Kathryn Schneider, 1,3,6,7,8 Brent E Hagel, $1,2,3,4,6,7,8$ Carolyn Emery. 'Sport Injury Prevention Research Centre, Faculty of Kinesiology, University of Calgary, Calgary, Canada; ${ }^{2}$ Hotchkiss Brain Institute, Cumming School of Medicine, University of Calgary, Calgary, Canada; ${ }^{3}$ Alberta Children's Hospital Research Institute, Cumming School of Medicine, University of Calgary, Calgary, Canada; ${ }^{4}$ Sport Medicine Centre, University of Calgary, Calgary, Canada; ${ }^{5}$ Evidence Sport and Spinal Therapy, Calgary, Canada; ${ }^{6}$ Community Health Sciences, Cumming School of Medicine, University of Calgary, Calgary, Canada; 'Department of Pediatrics, Cumming

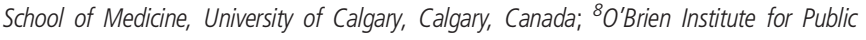
Health, Cumming School of Medicine, University of Calgary, Calgary, Canada
\end{abstract}

\subsection{6/bjsports-2021-IOC.75}

Background In 2004, a survey conducted in Alberta, Canada $(n=2850)$ reported that $93.8 \%$ of high school students (ages 14-19) participated in sport over the previous year with injury rates (IR) of 65.7 injuries/100 students/year, 40.2/100 students/year for injuries requiring medical attention and 49.9 injuries/100 students/year for time loss injuries. Over the past decade, the Sport Injury Prevention Research Centre has introduced injury prevention programs to decrease the risk of sport-related injury among adolescents in schools and the community.

Objective To examine sport participation and injury rates in high school students.

Design Cross-sectional survey.

Setting High schools (Alberta, Canada).

Participants High school students ( $\mathrm{n}=2029 ; 958$ male, 1048 female, 23 identified as 'other') from 24 of 63 (38\%) schools targeted for recruitment.

Assessment of Risk Factors Students completed a web-based survey during class (October 2018 -March 2019). Students identified the top 3 sports for participation in the past year.

Main Outcome Measurements Self-reported IR for 1) any sport-related injury over the last year, 2) most serious injury resulting in medical attention, and 3) most serious injury resulting in being restricted from sport $\geq$ one day adjusting for cluster by school.

Results Of the 2029 respondents, 861/958 (89.9\%) males, $886 / 1048(84.5 \%)$ females and $16 / 23(69.6 \%)$ of those who identified as 'other', participated in a sport/recreational activity in the last year. Of the 1971 students who completed the question on sport injury, 892 reported at least one injury over the last year ( $\mathrm{IR}=45.3$ injuries/100 students/ year (95\%CI, 40.1-50.8). The IR including only injuries resulting in medical attention was 27.8 injuries/100 students/year $(95 \% \mathrm{CI}, 23.4,32.7)$ and resulting in time loss from sport was 35.9 injuries/100 students/year (95\%CI, 40.4-41.8).

Conclusions The sport-related injury rate for adolescents in Alberta is lower than previously reported 10 years ago. While, the decrease may be associated with wide scale injury prevention initiatives, it may also be related to a decline in sport participation. Future studies evaluating injury prevention strategies broadly are necessary.

\section{THE PREVALENCE OF INDICATORS OF RELATIVE ENERGY DEFICIENCY IN SPORT (RED-S) IN AUSTRALIAN ELITE AND PRE-ELITE FEMALE ATHLETES}

\begin{abstract}
${ }^{1,2}$ Margot Rogers, ${ }^{1}$ Nicole Vlahovich, ${ }^{1}$ David Hughes, ${ }^{2}$ David Pyne, ${ }^{3}$ Shona Halson, ${ }^{2}$ Gregory Lovell, ${ }^{1,2}$ Renee Appaneal, ${ }^{4}$ Nic West, ${ }^{5}$ Bronwen Lundy, ${ }^{1}$ Marijke Welvaert, 1,5 Louise Burke, ${ }^{1,2}$ Gordon Waddington, ${ }^{1,2}$ Michael Drew. 'Australian Institute of Sport, Canberra, Australia; ' University of Canberra Research Institute for Sport and Exercise, Canberra, Australia; ${ }^{3}$ School of Behavioural and Health Sciences, Australian Catholic University, Brisbane, Australia; ${ }^{4}$ Mucosal Immunology Research Group, Griffith University, Gold Coast, Australia; ${ }^{5}$ Mary MacKillop Institute for Health Research, Australian Catholic University, Melbourne, Australia
\end{abstract}

\subsection{6/bjsports-2021-IOC.76}

Background Athlete health, training availability and performance can be impeded due to Relative Energy Deficiency in Sport (RED-S). Development of RED-S is underpinned by low energy availability (LEA), however its natural history is yet to be defined.

Objective To quantify the prevalence of issues previously described in association with RED-S in a mixed-sport cohort of female athletes, to inform the definition of its natural history.

Design This cross-sectional, observational study in athletes from eight sports utilised validated screening questionnaires and clinical investigations.

Setting Athletes were competing and/or training at a state, national or international level at the time of recruitment. Questionnaire data were collected electronically, whilst clinical investigations were undertaken at the Australian Institute of Sport.

Participants Females $\geq 15$ years old from the National Sporting Organisations approached by the researchers were eligible $(n=112)$.

Assessment of Risk Factors Psychology, nutrition and sleep screening questionnaires were administered. Fasted blood tests, indirect calorimetry, dual-energy $\mathrm{x}$-ray absorptiometry scans, and diagnostic psychiatric clinical interviews were undertaken.

Main Outcome Measurements Menstrual function, bone mineral density, thyroid function, resting metabolic rate, serum ferritin, mental health, lipid profile, gastrointestinal symptoms and recent illness and/or injury resulting in sports incapacity were assessed as indicators of the health consequences associated with RED-S.

Results Almost all (87\%) participants demonstrated at least one indicator of RED-S consequences, with $81 \%$ exhibiting between one and three indicators. Participants most commonly displayed impairments of the immunological (24\%), cardiovascular (28\%), haematological (29\%) and gastrointestinal (46\%) systems. Risk of LEA was identified in $11-55 \%$ of participants, and one third of the assessed cohort had a diagnosed psychiatric condition.

Conclusions RED-S consequences were prevalent.

These results have informed secondary prevention strategies whereby early detection of symptoms leads to early intervention prior to multi-system involvement. Further work is warranted to determine the interactions between these impairments, LEA and subsequent RED-S, to inform treatment and prevention. 